\title{
Manual Therapy for Cervicogenic Headache
}

\section{Umasankar Mohanty*}

Professor, Founder President, Manual Therapy Foundation of India ${ }^{\circledR}$, India

*Corresponding Author: Umasankar Mohanty, Professor, Founder President, Manual Therapy Foundation of India®, India.
Received: January 06, 2021

Published: July 14, 2021

(C) All rights are reserved by Umasankar

Mohanty.

\section{Abstract}

Headaches are often caused by disorders of the neck or physical and emotional tension. Skilled manipulative physiotherapists can successfully treat headaches originating from the neck or soft tissues and facilitate to prevent the pain arising from cervicogenic headache.

Keywords: Cervicogenic Headache; Manual Therapy; Neck

\section{Introduction}

Cervicogenic headache (CEH) is an unilateral headache localised in the neck or occipital region, projecting to the frontal and temporal regions. CEH has been described as early as 1926 by Barre M. Sjaastad., et al. were the first to give its current name in 1998. The cervicogenic headache accounts for up to $20 \%$ of all headaches (Pfaffenrath and Kaube 1990; Maciel, 1997).

\section{Presentations of cervicogenic headache}

The diagnostic criteria for cervicogenic headache as outlined by Sjaastad., et al. (1998) and the International Headache Society [1] are principally based on subjective characteristics. There is an established link between impairment in cervical joints and cervicogenic headache pathogenesis (Jull., et al. 1988; Jaeger, 1989; Treleaven., et al. 1994). The following are the criterias.

- Altered neck posture or restricted cervical range of motion (Hall, Robinson).

- The head pain triggered by active neck movement, passive neck positioning (extension or extension with rotation toward the side of pain, or on applying digital pressure to the involved facet regions or over the ipsilateral greater occipital nerve (Sjaastad 1998).

- Muscular trigger points are usually found in the suboccipital, cervical, and shoulder musculature, and these trigger points can also refer pain to the head when manually or physically stimulated (Sjaastad 1998).

- There are no neurologic findings of cervical radiculopathy, though the patient might report scalp paresthesia or dysesthesia (Sjaastad 1998).

- Diagnostic imaging such as radiography, magnetic resonance imaging (MRI), and CT are normal.

As the headaches can be felt due to the Migraine and the Cervicogenic headache in following I am differentiating between the two in the table.

\section{Mechanism of cervicogenic headache}

The trigeminocervical nucleus is a region of the upper cervical spinal cord where sensory nerve fibers in the descending tract of the trigeminal nerve (trigeminal nucleus caudalis) are believed to interact with sensory fibers from the upper cervical roots. This 


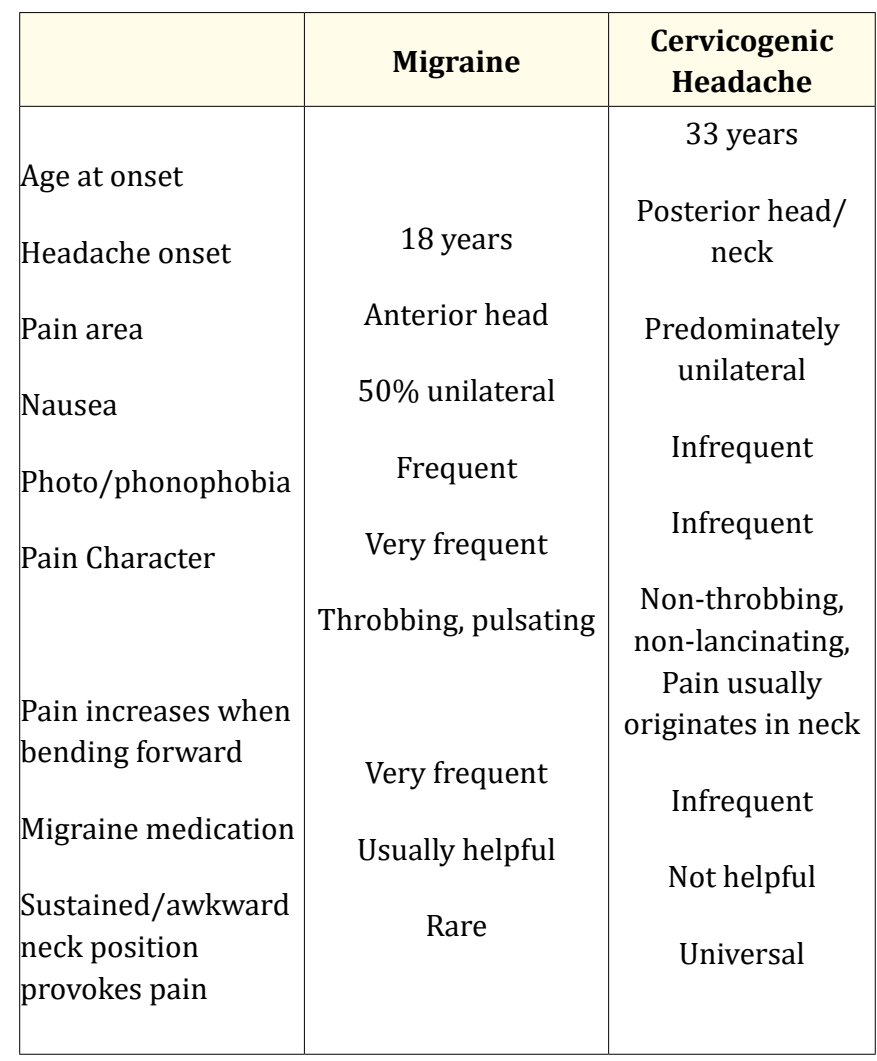

Table 1: Summary of subjective diagnostic criteria contrasting Migraine and Cervicogenic Headache.

functional convergence of upper cervical and trigeminal sensory pathways allows the bidirectional referral of painful sensations between the neck and trigeminal sensory receptive fields of the face and head. A functional convergence of sensorimotor fibers in the spinal accessory nerve (CN XI) and upper cervical nerve roots ultimately converge with the descending tract of the trigeminal nerve and might also be responsible for the referral of cervical pain to the head (Biondi 2005) figure 1.

The structures with possibilities of giving rise to the Cervicogenic Headaches are the upper cervical nerves (greater and lesser occipital nerves), nerve roots, cervical muscles, cervical discs and zygapophyseal (facet) joints, and atlantoaxial and atlantooccipital joints. Cervicogenic headache may arise not only from the upper, but also from the middle and even from the lower cervical area (Sjaastad O., et al. 1998).

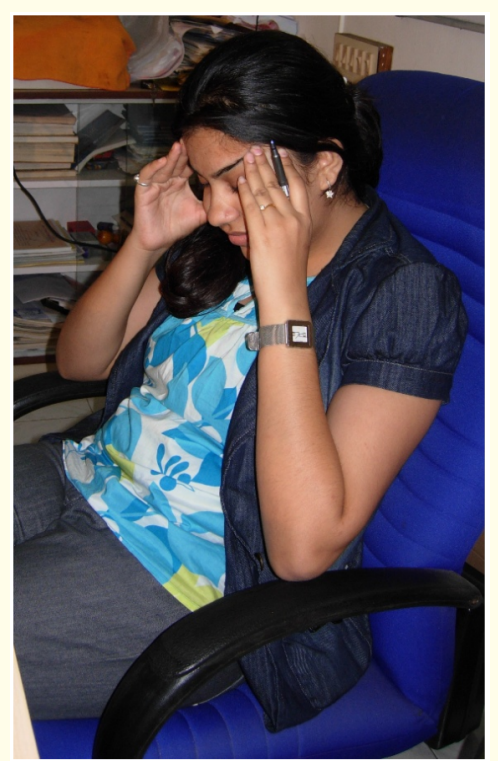

Figure 1

Predisposing factors

- Working with head down or to one side for long periods.

- Sleeping in Awkward positions.

- $\quad$ king with Hunching shoulders.

- Assuming poked chin posture.

- Regular clenching of teeth.

\section{Assessment of Cervicogenic Headache}

A range of examination procedures, (active and passive motions test) have been described to determine the presence of upper cervical spine joint dysfunction (Greenman, 1996; Maitland., et al. 2001; Monaghan, 2001). Such manual examination procedures have been shown to detect symptomatic cervical joint dysfunction in a number of studies of cervicogenic headache (Jaeger,1989; Jensen., et al. 1990; Watson and Trott, 1993; Drefus., et al. 1994; Treleaven., et al. 1994; Whittingham., et al. 1994; Schoensee., et al. 1995). The sequence of assessment are as follows.

- Subjective Examination

- Observation

- Active Movements with Overpressures 
- Palpation (Superficial palpation for soft tissues and Deep palpation for Joints)

- $\quad$ Trigger point palpation and muscle length testing.

- Passive Physiological Intervertebral Movements (PPIVM's)

- Passive Accessory Intervertebral Movements (PAIVM's)

Manual therapy management for cervicogenic headache

The following manual therapy techniques can be used to alleviate the symptoms of cervicogenic headache and can give complete relief from pandemonium of cervicogenic headache.

\section{Cranial base release}

The subject is in supine lying position. The therapist places the pulp of the 4 fingers( except the thumb) at the occiput. The therapist applies the pressure directed postero-superior till the barrier is felt. The therapist applies the pull in the paraphysiological space 4-6 times. This technique helps to release the suboccipital muscle rectus capitis posterior minor, which is more prone to develop tightness and trigger point.
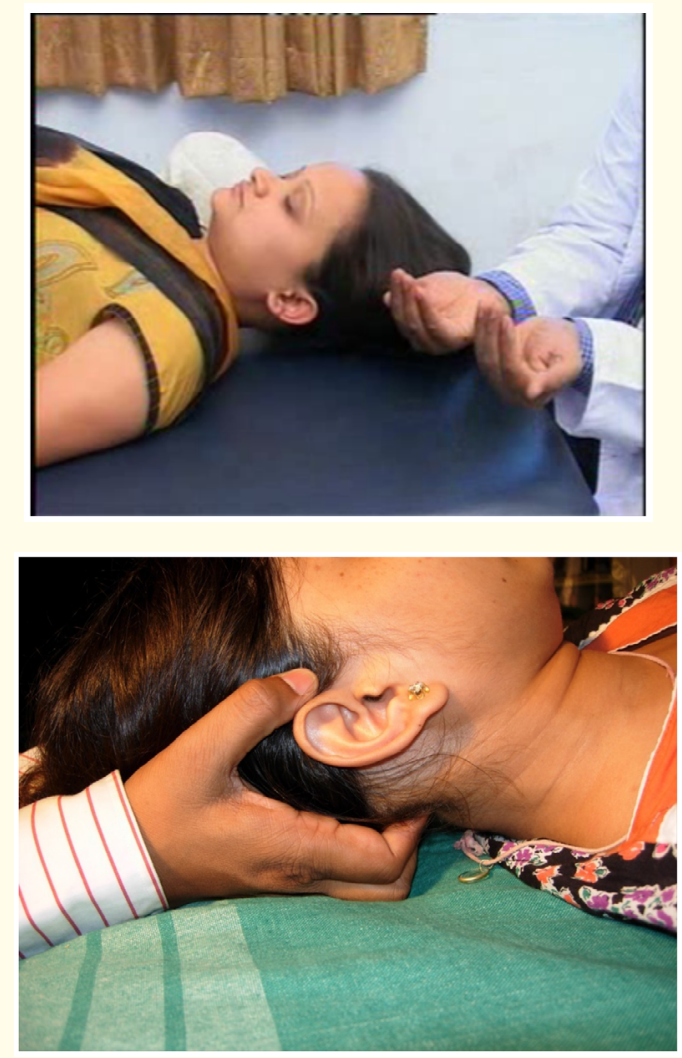

Figure 2

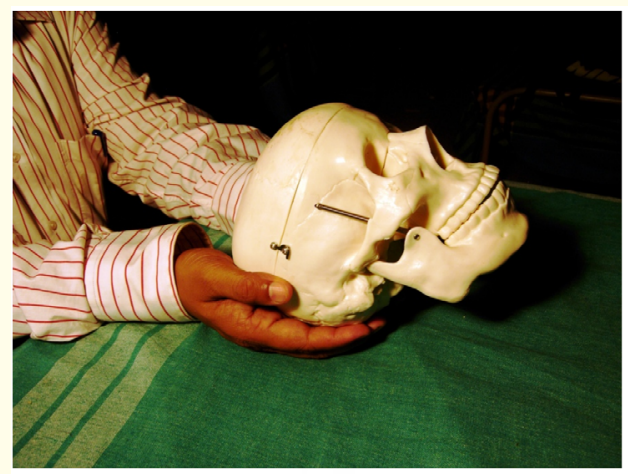

Figure 2a

\section{Cervical A-P Glide}

The subject is in supine lying position. The therapist supports the occiput of the subject with one hand and the other hand index and mid finger are placed anterior to the mantle region. Then the therapist gives anterior to posterior translation for about 6-10times. This technique is useful with the subjects with poked chin and it helps to release the periarticular structures of the upper cervical region.

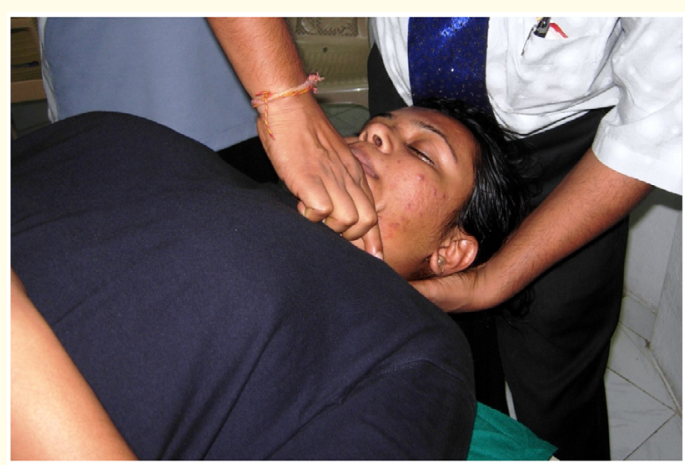

Figure 3

\section{Lateral P-A Glide}

The subject is in prone lying position. The therapist palpates for the lateral joint of the affected segment and applies posterior to anterior glide. This technique facilitates to release the lateral joint of CO-C1 region, C1-C2 region or the Zygapophyseal joint below $\mathrm{C} 2$. 


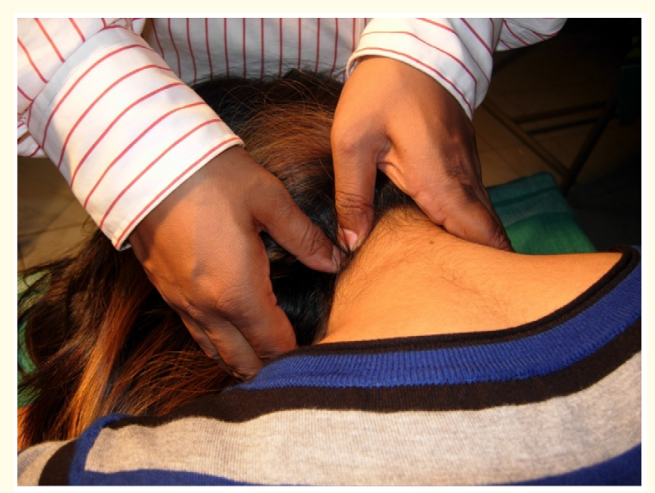

Figure 4

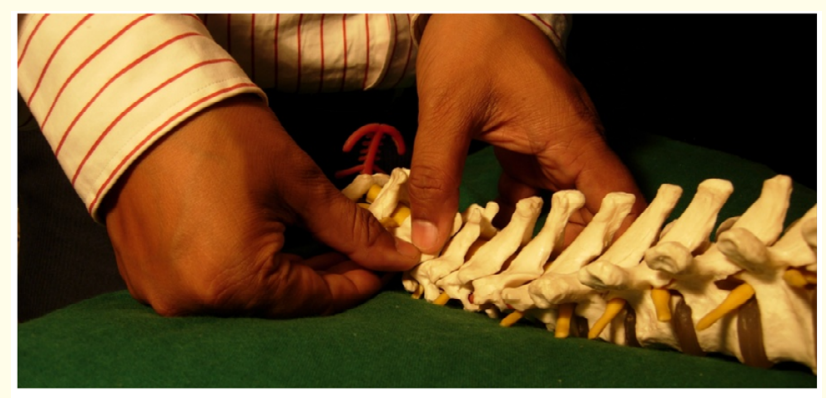

Figure 4a

Transverse glide

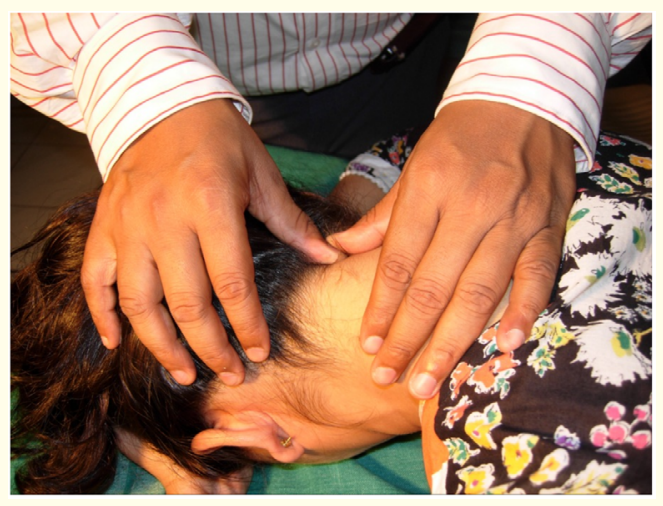

Figure 5

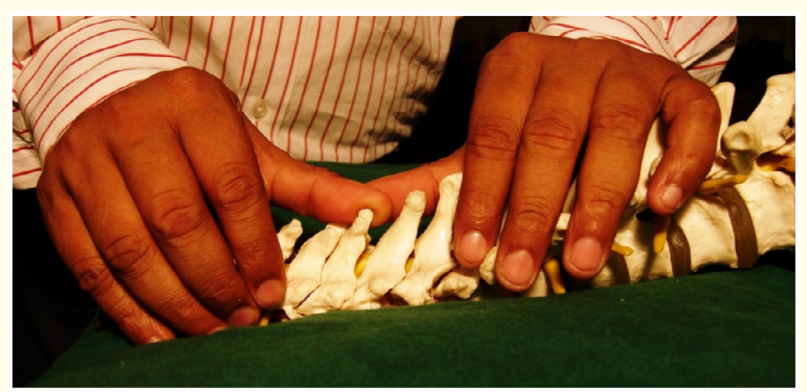

Figure 5a

The subject is in prone lying position. The therapist places the pulp of the thumb is placed at lateral aspect of the spinous process, thumb of the other hand reinforces the thumb in contact. The glides are applied for about 10 times. The technique is useful if there is rotation around the y-axes and the technique is applied opposite to the side of rotation so that it returns to the neutral position.

High velocity low amplitude thrust techniques (HVLAT)

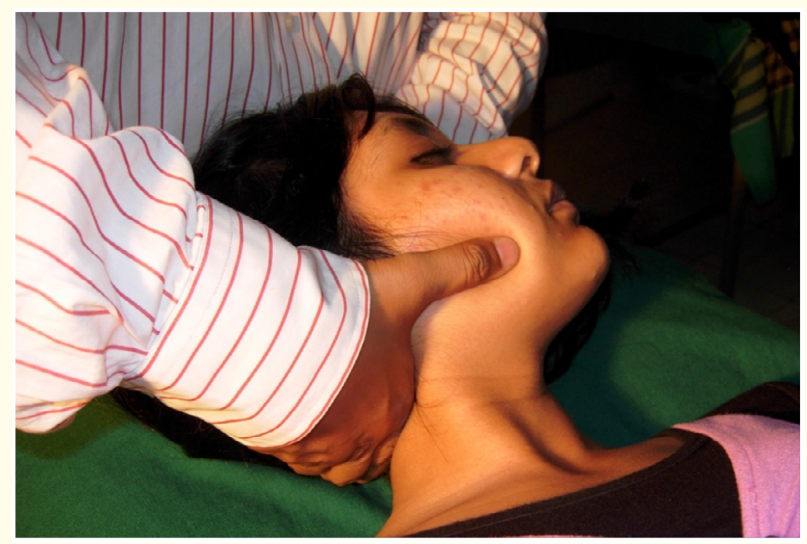

Figure 6

For the lateral joint HVLAT techniques can be applied with adjustment of adequate leverage. The neck is taken for rotation to opposite side, side flexion to same side and from the articular pillar thrust is applied. The direction of the thrust can be upslope towards the opposite side eye ball or can be downslope towards the opposite shoulder depeneing on the dysfunction. 
Muscle energy techniques for suboccipitals

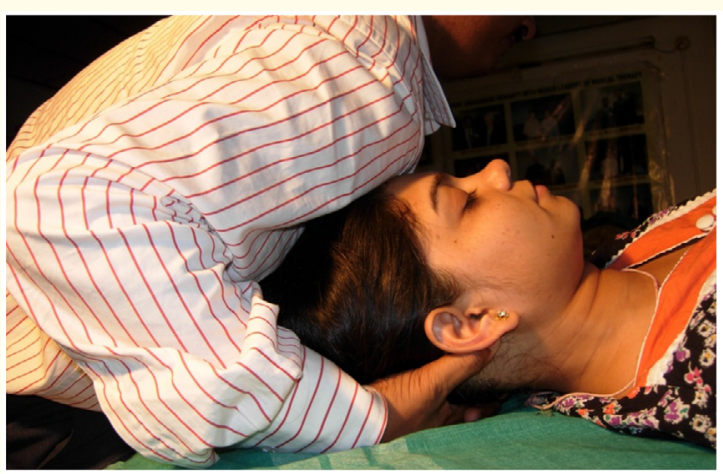

Figure 7

The subject is in supine lying. The therapist supports the occiput with one hand. The therapist places the ventral aspect of his/her shoulder at anterior aspect of the forehead. The therapist reaches the barrier by passively tucking the chin in. The subject is asked to poke the chin against the resistance( performing isometric contraction of the suboccipitals and held for about 6 seconds with one third of the muscular effort.

If there are existing trigger points of trapezius and sternocleidomastoid then Muscle Energy Techniques can be applied for the muscles [2-8].

\section{Conclusion}

To conclude cervicogenic headache can be very effectively tackled with manual therapy techniques described above. It is very important for the therapist to identify the problem by proper clinical reasoning and then to apply the techniques judiciously.

\section{Bibliography}

1. International Headache Society. "The International Classification of Headache Disorders". 2nd ed. Cephalalgia 24 (2004): 9-160.

2. Stovner L., et al. "The global burden of headache: A documentation of headache prevalence and disability worldwide". Cephalalgia 27 (2007): 193-210.

3. Diener I. "The impact of cervicogenic headache on patients attending a private physiotherapy practice in Cape Town". South
African Journal of Physiotherapy 57 (2001): 35-39.

4. Lipton RB and Stewart WF. "The epidemiology of migraine". European Neurology 34 (1994): 6-11.

5. Hu H., et al. "Burden of migraine in the United States: Disability and economic costs". Archives of Internal Medicine 1 (1999): 813-818.

6. Fishbain DA., et al. "International Headache Society headache diagnostic patterns in pain facility patients". Clinical Journal of Pain 17 (2001): 78-93.

7. Haldeman S and Dagenais S. "Cervicogenic headaches: A critical review". Spine Journal 1 (2001): 31-46.

8. Sjaastad $\mathrm{O}$ and Bakketeig LS. "Prevalence of cervicogenic headache: Vaga study of headache epidemiology". Acta Neurologica Scandinavica 117 (2008): 170-183.

\section{Volume 4 Issue 8 August 2021}

(C) All rights are reserved by Umasankar Mohanty. 